NASA/CR—2007-214671

\title{
Progress Toward Improving Jet Noise Predictions in Hot Jets
}

Abbas Khavaran

ASRC Aerospace Corporation, Cleveland, Ohio

Donald C. Kenzakowski

Combustion Research \& Flow Technology, Inc., Pipersville, Pennsylvania 


\section{NASA STI Program . . . in Profile}

Since its founding, NASA has been dedicated to the advancement of aeronautics and space science. The NASA Scientific and Technical Information (STI) program plays a key part in helping NASA maintain this important role.

The NASA STI Program operates under the auspices of the Agency Chief Information Officer. It collects, organizes, provides for archiving, and disseminates NASA's STI. The NASA STI program provides access to the NASA Aeronautics and Space Database and its public interface, the NASA Technical Reports Server, thus providing one of the largest collections of aeronautical and space science STI in the world. Results are published in both non-NASA channels and by NASA in the NASA STI Report Series, which includes the following report types:

- TECHNICAL PUBLICATION. Reports of completed research or a major significant phase of research that present the results of NASA programs and include extensive data or theoretical analysis. Includes compilations of significant scientific and technical data and information deemed to be of continuing reference value. NASA counterpart of peer-reviewed formal professional papers but has less stringent limitations on manuscript length and extent of graphic presentations.

- TECHNICAL MEMORANDUM. Scientific and technical findings that are preliminary or of specialized interest, e.g., quick release reports, working papers, and bibliographies that contain minimal annotation. Does not contain extensive analysis.

- CONTRACTOR REPORT. Scientific and technical findings by NASA-sponsored contractors and grantees.
- CONFERENCE PUBLICATION. Collected papers from scientific and technical conferences, symposia, seminars, or other meetings sponsored or cosponsored by NASA.

- SPECIAL PUBLICATION. Scientific, technical, or historical information from NASA programs, projects, and missions, often concerned with subjects having substantial public interest.

- TECHNICAL TRANSLATION. Englishlanguage translations of foreign scientific and technical material pertinent to NASA's mission.

Specialized services also include creating custom thesauri, building customized databases, organizing and publishing research results.

For more information about the NASA STI program, see the following:

- Access the NASA STI program home page at http://www.sti.nasa.gov

- E-mail your question via the Internet to help@sti.nasa.gov

- Fax your question to the NASA STI Help Desk at 301-621-0134

- Telephone the NASA STI Help Desk at 301-621-0390

- Write to: NASA Center for AeroSpace Information (CASI) 7115 Standard Drive Hanover, MD 21076-1320 
NASA/CR—2007-214671

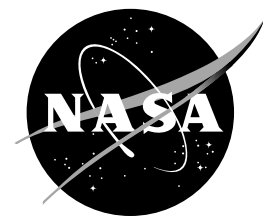

\section{Progress Toward Improving Jet Noise Predictions in Hot Jets}

Abbas Khavaran

ASRC Aerospace Corporation, Cleveland, Ohio

Donald C. Kenzakowski

Combustion Research \& Flow Technology, Inc., Pipersville, Pennsylvania

Prepared for the

45th Aerospace Sciences Meeting and Exhibit

sponsored by the American Institute of Aeronautics and Astronautics

Reno, Nevada, January 8-11, 2007

Prepared under Contract NNC06BA07B

National Aeronautics and

Space Administration

Glenn Research Center

Cleveland, Ohio 44135 


\section{Acknowledgments}

The authors are grateful to Dr. James Bridges, Acoustics Branch, NASA Glenn Research Center, for providing the acoustic data.

This work was sponsored by the Fundamental Aeronautics Program at the NASA Glenn Research Center.

Level of Review: This material has been technically reviewed by expert reviewer(s).

Available from

NASA Center for Aerospace Information 7115 Standard Drive

Hanover, MD 21076-1320
National Technical Information Service 5285 Port Royal Road Springfield, VA 22161 


\title{
Progress Toward Improving Jet Noise Predictions in Hot Jets
}

\author{
Abbas Khavaran \\ ASRC Aerospace Corporation \\ Cleveland, Ohio 44135 \\ Donald C. Kenzakowski \\ Combustion Research \& Flow Technology, Inc. (CRAFT Tech) \\ Pipersville, Pennsylvania 18947
}

\begin{abstract}
An acoustic analogy methodology for improving noise predictions in hot round jets is presented. Past approaches have often neglected the impact of temperature fluctuations on the predicted sound spectral density, which could be significant for heated jets, and this has yielded noticeable acoustic under-predictions in such cases. The governing acoustic equations adopted here are a set of linearized, inhomogeneous Euler equations. These equations are combined into a single third-order linear wave operator when the base flow is considered as a locally parallel mean flow. The remaining second-order fluctuations are regarded as the equivalent sources of sound and are modeled. It is shown that the hot jet effect may be introduced primarily through a fluctuating velocity/enthalpy term. Modeling this additional source requires specialized inputs from a RANS-based flowfield simulation. The information is supplied using an extension to a baseline two-equation turbulence model that predicts total enthalpy variance in addition to the standard parameters. Preliminary application of this model to a series of unheated and heated subsonic jets shows significant improvement in the acoustic predictions at the $90^{\circ}$ observer angle.
\end{abstract}

\section{Introduction}

This paper discusses application of an improved physics-based methodology in predicting jet noise for heated subsonic jets, with model extensibility for future predictions of supersonic heated jets. The goal is to develop a methodology that accurately computes the far-field sound for jets at all observer angles and all operating conditions. In order to achieve this goal, efforts are ongoing at NASA Glenn Research Center to examine the current theories in jet noise and to combine that with the best practices in numerical modeling, all of which must be validated by experiments. Physics-based jet noise predictions codes such as JeNo (ref. 1) that depend on their source modeling, as well as mean flow and turbulence information from RANS codes, are inevitably going to be sensitive to the details of the modeling and the accuracy of their RANS input. JeNo, which is still in its development phase at NASA Glenn Research Center, has been validated for unheated axisymmetric jets at subsonic and low supersonic conditions. Sample calculations at heated conditions, however, point to a noticeable under-prediction relative to measurements.

To motivate the discussion, consider the $90^{\circ}$ jet noise predictions at the Tanna test conditions SP07and SP49 reference 2, i.e., stagnation temperature ratio of 1.0 and 3.14 (see table II). The predictions (ref. 1) use the so-called self-noise source term in the acoustic modeling (eq. (B5), appendix B), and RANS input from the NASA Wind code. As shown in figure 1, hot jet spectral predictions tend to be significantly under-predicted with respect to acoustic data. Moreover, this observation applies to predictions using CFD inputs that otherwise provide good agreement with experimental PIV data for both mean and fluctuating velocity in the developing jet region, where most of the noise is produced. For example, a detailed CFD study was conducted utilizing available PIV data measured in the developing jet region for a range of jet exit velocities and temperatures. Findings (ref. 3) indicate that the turbulent kinetic energy levels and peak centerline locations predicted by a standard high Reynolds number variant for the $\mathrm{k}-\varepsilon$ model trend quantitatively well with increased jet temperature. In addition, mean flow mixing appears well captured without the need for additional modifications to enhance shear layer mixing growth due to density gradients. However, as seen in figure 1, the predicted changes in the turbulence values are insufficient to account for the peak noise differences between heated and unheated jets. Consequently, it appears plausible to attribute the noise prediction deficit to heat-related sources. 


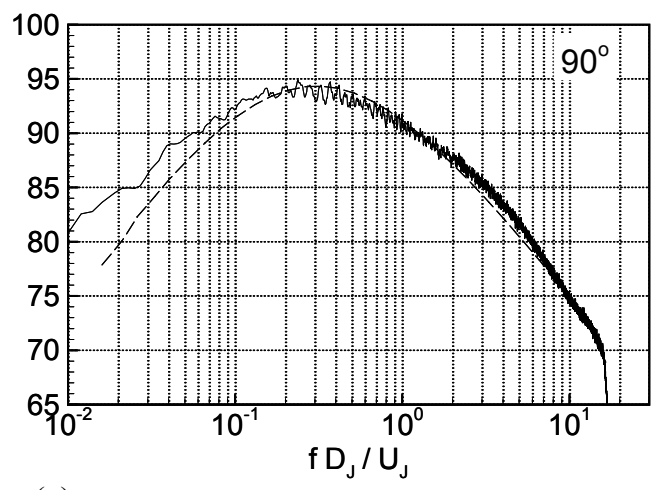

(a)

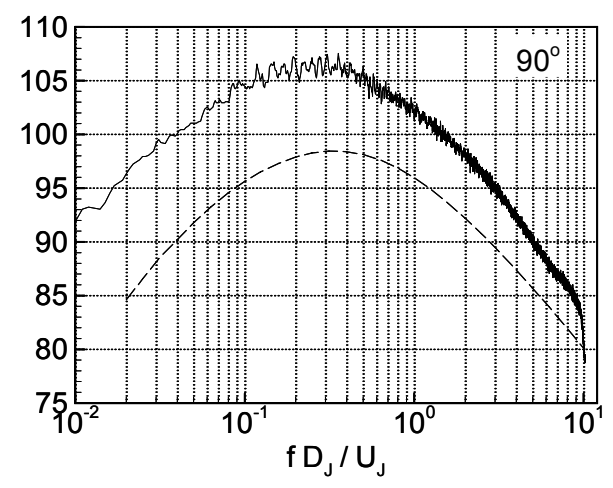

(b)

Figure 1. - Spectra/data comparisons $(\mathrm{dB})$ using baseline JeNo code for subsonic jet cases at $90^{\circ}$ inlet angle: (a) SP07 (unheated) and (b) SP49 (heated)(ref. 1).

Noise from heated jets is of particular interest in aeroacoustics due to its far-reaching implications in defining the source. Lighthill's definition of equivalent aerodynamic noise sources $T_{i j}=\rho v_{i} v_{j}+\left(p-c_{\infty}^{2} \rho\right) \delta_{i j}-\tau_{i j}$ has been a subject of wide-spread scrutiny and interpretations. This has led to scaling laws that relate the far-field sound to flow parameters such as velocity and temperature in a variety of forms. In the absence of viscous stresses $\tau_{i j}$, the momentum flux term $\partial^{2} \rho v_{i} v_{j} / \partial^{2} x_{i} x_{j}$ is usually considered as the primary source of sound in isothermal jets. Heat addition has a multitude of effects on noise that depends on the jet velocity, frequency, and angle. Experimental observations (refs. 2, 4, and 5) concur that at high speeds, heat addition results in noise reduction at all frequencies, while at low speeds, say $U_{j} / c_{\infty}=0.50$ or below, it amplifies the low to mid frequency jet noise with minimal effect at the high frequency. In between, say at $U_{j} / c_{\infty} \sim 0.70$, the spectrum shows a cross-over relative to the unheated case that indicates an enhancement of the low-frequency noise and slight weakening of the high-frequency amplitude.

These observations have compelled a number of researchers to examine the so-called pressure/density term $\left(p-c_{\infty}^{2} \rho\right)$ as a potential additional source and formulate scaling laws that resemble the usual $U^{8}$ law suggested based on the first source term. A recent noise data analysis (ref. 6), examines a more general power law $A U^{n}$ where amplitude $A$ and power $n$ are parameters that depend on angle and jet temperature. Morfey et al. (ref. 7) revised Lush and Fisher's (ref. 8) version of entropy source term and offered a new two-scaling hypothesis, in favor of $U^{6}$ and $U^{4}$ power laws, and with some dependence on the mean temperature gradient. Additionally, they prompted to account for the mean flow effects using some form of Geometric Acoustic approximation. Lilley (ref. 9) proposed a second order wave equation for the pressure fluctuations, similar to Lighthill's, but replaced with its equivalent from the energy equation. His new contribution to the total acoustic power consisted of a Dipole term with $U^{6}$ dependency, which also multiplied the variance of the total enthalpy. He provided some estimate of the relevance of the new source term without actually calculating the required enthalpy fluctuations.

The predictions provided in the current study are based on a more systematic form of the governing equations that separates the background flow from the fluctuations starting from the set of Euler equations. Following an analysis similar to Goldstein (ref. 10), and with suitable assumptions about the base flow, these equations are combined to form a third-order linear wave operator with pressure fluctuations $p^{\prime}$ as its dependent variable, and a set of non-linear terms that are second-order in fluctuating variables and are identified as the equivalent sources of sound. As seen in the following discussions, the analysis seems to point, among other factors, to a combination of fluctuating velocity/enthalpy term as an additional source.

To improve the RANS-based modeling capability for noise, a set of transport equations is utilized that predict the total temperature (or enthalpy) fluctuations in addition to the usual turbulence-related parameters. The approach, as discussed in (ref. 11), makes use of a baseline k- $\varepsilon$ turbulence model demonstrated in past work to predict correct mean flow velocity mixing, turbulent kinetic energy, and Reynolds stress variations for heated and unheated subsonic round jets. A generalized two-equation scalar variance model is available within this turbulence model framework, which utilizes an independent dissipation rate equation as well as the locally available turbulent velocity 
and time-scale information. Determination of the modeled scalar variance variable is principally achieved by selecting the appropriate mean flow quantity gradient for its production source term. In the past, this model has been used to predict the local variations in the turbulent Prandtl number and to study its impact on jet thermal mixing using an energy variance approach that tracked the variance in static temperature. The current study traces the variance of the total enthalpy instead as the parameter of interest for subsequent jet noise predictions. The difference between these two parameters becomes increasingly obvious with jet speed, producing a relatively noticeable static temperature variance within the jet shear layer even under unheated conditions compared to practically insignificant total temperature variance. It is interesting to note that, at least for the jet cases studied, the use of total enthalpy variance does not significantly alter predictions for the variable turbulent Prandtl number. The local turbulent thermal diffusivity is partially determined from the thermal time-scale; the predicted values for the variance dissipation rate are also affected proportionately by the scalar variance production term selection.

The remainder of this paper is divided into the following sections. The governing acoustic equations and the sources of jet noise in the specialized case of a locally parallel mean flow are presented. This builds grounds for modifications subsequently introduced into the transport equations that accommodate the flowfield information required for noise prediction. A summary of the baseline scalar variance model framework is presented first. Details regarding the model development can be found in (refs. 11 and 12). The variances in total and static temperatures are then compared for a series of heated and unheated subsonic jets. Comparative results for the subsonic jet cases feature significant improvements in predicted jet noise for heated jets. Discussions are currently restricted to the $90^{\circ}$ observer angle; results for other observer angles will be forthcoming in a future paper.

Extension of the modeling methodology to supersonic jets is still exploratory. Most flowfield modeling work related to jet noise in this area has focused principally on obtaining correct mean flow velocity and/or temperature decay rate predictions relative to available measurements. The experimentally observed longer potential core lengths for supersonic unheated jets, which decrease as the exit temperature is increased, have prompted use of compressibility corrections to the standard turbulent kinetic energy equation for best agreement with data. However, a physics-based jet noise prediction methodology additionally requires accurate portrayal of the underlying turbulence quantities in the developing jet region, and this remains an area where further research is needed. In the present study, we restrict our study of supersonic jets to highlight desired numerical sensitivity results for total enthalpy fluctuations in the mixing region. Further efforts for supersonic jets will require studying the impact of turbulent compressibility corrections on the predicted jet noise outcome and may likely require use of both experimental and numerical (using LES) findings as guidance.

\section{Acoustic Equations}

This section briefly describes the governing acoustic equation, which can best be described as a form of Lilley acoustic analogy in a locally parallel mean flow. The details of the source modeling and source/Green's functions convolution integral are given in appendices A and B.

Assuming that the ideal gas law $p=\rho \Re T$ holds, each flow variable is divided into its mean and fluctuating components

$$
p=\bar{p}+p^{\prime}, \quad \rho=\bar{\rho}+\rho^{\prime}, \quad v_{i}=\tilde{v}_{i}+v_{i}^{\prime}, \quad h=\tilde{h}+h^{\prime},
$$

where the over-bar is used to denotes a time-averaged value, and tilde is a Favre-averaged (i.e., mass-averaged) quantity, and the stagnation enthalpy is

$$
h_{t}=h+\frac{1}{2} v^{2}, \quad \tilde{h}_{t}=\tilde{h}+\frac{1}{2} \tilde{v}^{2}, \quad h_{t}^{\prime}=h^{\prime}+\frac{1}{2} v^{\prime 2} .
$$

The set of Euler's equations are now linearized using the above definitions, and expressed as two sets of equations governing the base flow and its fluctuations or residuals (ref. 10). Additional simplification is achieved only when isentropic form of the energy equation is considered and the base flow is described as a locally parallel mean flow

$$
\tilde{v}_{j}=\delta_{j 1} U\left(x_{2}, x_{3}\right), \quad \bar{\rho}=\bar{\rho}\left(x_{2}, x_{3}\right), \quad \bar{p}=\text { constant }
$$

The governing equations are thus combined into a single third-order wave equation

$$
L \pi^{\prime}=\Gamma, \quad \pi^{\prime} \equiv \frac{p^{\prime}}{\gamma \bar{p}}
$$


where the linear operator $L$ is

$$
\begin{gathered}
L f \equiv D\left(D^{2}-\frac{\partial}{\partial x_{j}} \tilde{c}^{2} \frac{\partial}{\partial x_{j}}\right) f+2 \tilde{c}^{2} \frac{\partial U}{\partial x_{j}} \frac{\partial^{2} f}{\partial x_{1} \partial x_{j}} \\
D \equiv \frac{\partial}{\partial t}+U \frac{\partial}{\partial x_{1}}
\end{gathered}
$$

and the source is given as

$$
\begin{aligned}
\Gamma & \cong D\left(\frac{\partial^{2} v_{i}^{\prime} v_{j}^{\prime}}{\partial x_{i} \partial x_{j}}-\frac{1}{2} D^{2}\left(\frac{v^{\prime 2}}{\tilde{h}}\right)\right) \\
& -2 \frac{\partial U}{\partial x_{j}}\left(\frac{\partial^{2} v_{i}^{\prime} v_{j}^{\prime}}{\partial x_{1} \partial x_{i}}+\frac{1}{2} D^{2}\left(\frac{v_{1}^{\prime} v_{j}^{\prime}}{\tilde{h}}\right)+\left(\frac{1}{\bar{\rho}} \frac{\partial \bar{\rho}}{\partial x_{i}}\right) \frac{\partial}{\partial x_{1}}\left(v_{i}^{\prime} v_{j}^{\prime}\right)\right) \\
& -D\left(D \frac{\partial}{\partial x_{j}}\left(v_{j}^{\prime} \frac{h_{t}^{\prime}}{\tilde{h}}\right)-\frac{\partial}{\partial x_{j}}\left(\frac{v_{i}^{\prime} v_{j}^{\prime}}{\bar{\rho}} \frac{\partial \bar{\rho}}{\partial x_{i}}\right)\right) .
\end{aligned}
$$

In view of the ideal gas law, we require

$$
\bar{\rho} \tilde{h}=\frac{\gamma}{\gamma-1} \bar{p}, \quad \gamma \bar{p}=\bar{\rho} \tilde{c}^{2} .
$$

Three pairs of brackets in eq. (7) designate three distinct sources. The former terms in each bracket are identified as self, shear, and enthalpy sources respectively. Self and shear source terms are second-order in fluctuating velocity, and are each complemented by a second term involving the mean flow enthalpy which is not zero even when the flow is isothermal, but may be considered small relative to the former. For example, sample predictions of (ref. 13)

conclude that $\frac{\partial U}{\partial x_{j}} D^{2}\left(\frac{v_{1}^{\prime} v_{j}^{\prime}}{\tilde{h}}\right)$ is small relative to other terms in unheated jets. Source terms that consist of the mean density gradient are negligible in unheated jets, but could potentially become important as jets get hot. A detailed comparison of the individual source terms of eq. (7) will be presented in an upcoming paper for all set points shown in table II. It turns out that the major difference between the unheated and heated jets is due to the fluctuations in stagnation enthalpy that appears in the third bracket above. This term is first order in velocity fluctuations and its radiated sound power dominates that of density gradient term as well as the self-noise source component as jets become hot.

Spectral prediction presented in section VII display the two relatively more significant source terms denoted as self- and enthalpy-noise, and are based on eqs. (B5) and (B6) discussed in the appendix (see appendix A for source modeling and appendix $\mathrm{B}$ for the derivation of the spectral functions).

\section{Enthalpy Variance and Dissipation Rate Model}

The high-Reynolds-number form of the equations governing the transport of the total enthalpy variance and its dissipation rate is based in concept on the work of Nagano and Kim (ref. 12) for generalized turbulent thermal diffusivity

$$
\begin{gathered}
\frac{D}{D t} \rho k_{t}=\frac{\partial}{\partial x_{j}}\left[\left(\alpha+\frac{\alpha_{t}}{\sigma_{k t}}\right) \frac{\partial k_{t}}{\partial x_{j}}\right]+P_{k_{t}}-2 \rho \varepsilon_{t}, \\
\frac{D}{D t} \rho \varepsilon_{t}=\frac{\partial}{\partial x_{j}}\left[\left(\alpha+\frac{\alpha_{t}}{\sigma_{\varepsilon t}}\right) \frac{\partial \varepsilon_{t}}{\partial x_{j}}\right]+\left[\left(\frac{\varepsilon_{t}}{k_{t}}\right)\left(C_{d 1} \frac{P_{k_{t}}}{\rho \varepsilon_{t}}-C_{d 2}\right)+\left(\frac{\varepsilon}{k}\right)\left(C_{d 3} \frac{P_{k}}{\rho \varepsilon}-C_{d 4}\right)\right] \rho \varepsilon_{t} .
\end{gathered}
$$


Here, $k$ and $\varepsilon$ denote the turbulent kinetic energy (TKE) and dissipation rate obtained from a companion twoequation turbulence model, $\alpha$ and $\alpha_{t}$ are the molecular and eddy diffusivities for heat, and $P_{k}$ is the production term used in the TKE equation. Values utilized for the modeling constants in the variance dissipation rate equation are based on results described in reference 11 and are listed in table I.

TABLE I.-ENERGY VARIANCE DISSIPATION RATE COEFFICIENT CONSTANTS

\begin{tabular}{|c|c|c|c|c|c|}
\hline$C_{d 1}$ & $C_{d 2}$ & $C_{d 3}$ & $C_{d 4}$ & $\sigma_{k t}$ & $\sigma_{\varepsilon t}$ \\
\hline 2.0 & 0.72 & 2.2 & 0.8 & 1.5 & 1.5 \\
\hline
\end{tabular}

Physical interpretation of the quantity $k_{t}$ tracked within the CFD simulation is based on selection of the flow variable gradient used for the turbulent variance production term $P_{k_{t}}$ For example, Nagano and Kim originally solved directly for the static temperature variance. Recent numerical studies modeling helium jets and afterburning rockets plumes indicate improved model generalization using an internal energy variance approach. As shown below, further extension of the framework to model the total enthalpy variance was found necessary to improve jet noise prediction.

Two modeling approaches were studied for use in hot jet noise prediction. The first utilized the internal energy variance as described in reference 11 . The second modeled the total enthalpy variance, with appropriate variable substitution for the variance dissipation rate equation. The production term is then rewritten for the selected method as follows:

$$
\begin{array}{ll}
P_{k_{t}}=2 \alpha_{t} \frac{\partial e}{\partial x_{j}} \frac{\partial e}{\partial x_{j}}, & \quad \text { (internal energy variance) } \\
P_{k_{t}}=2 \alpha_{t} \frac{\partial h_{t}}{\partial x_{j}} \frac{\partial h_{t}}{\partial x_{j}}, & \quad \text { (total enthalpy variance) }
\end{array}
$$

Where $e$ is the specific internal energy and $h_{t}$ is the specific total enthalpy. For a calorically and thermally perfect gas, the specific heat constants are invariant with temperature, and so the temperature variance can be expressed as follows:

$$
\begin{aligned}
& k_{t}=\overline{e^{\prime} e^{\prime}}=c_{v}{ }^{2}\left(\overline{T^{\prime} T^{\prime}}\right), \quad \text { (internal energy variance) } \\
& k_{t}=\overline{h_{t}^{\prime} h_{t}^{\prime}}=c_{p}{ }^{2}\left(\overline{T_{t}^{\prime} T_{t}^{\prime}}\right), \quad \text { (total enthalpy variance) }
\end{aligned}
$$

and $c_{p}=\gamma c_{v}$. Values for the coefficient constants in the variance dissipation rate equation for both approaches were unchanged.

When extending the variance model to predict thermal eddy diffusivity, an expression for the thermal turbulent time scale is needed. Based on results from past work, the following expression was used:

$$
\tau_{t}^{2}=\left(\frac{k}{\varepsilon}\right) \max \left[\left(\frac{k_{t}}{\varepsilon_{t}}\right),\left(\frac{C_{\mu}}{C_{\lambda}}\right)\left(\frac{k}{\varepsilon}\right)\right], \quad C_{\mu}=0.09
$$

The thermal eddy diffusivity, applied to the turbulent diffusion term in the mean energy equation and the variance production term, is written for model closure as

$$
\alpha_{t}=C_{\lambda} \rho k \tau_{t}
$$

As recommended by Chidambaram et al. (ref. 14), value of $C_{\lambda}=0.14$ was used. The local turbulent Prandtl number is then defined as the ratio of the eddy viscosity to the thermal eddy diffusivity

$$
\operatorname{Pr}_{t}=\mu_{t} / \alpha_{t} .
$$




\section{Subsonic Round Jet Flowfield Simulations}

Both the energy variance and total enthalpy variance models were applied to the simulations of several subsonic round jet cases, with nozzle exhaust conditions defined by set points within the Tanna matrix. A summary of these cases is listed in table II. In past work, these cases were studied extensively to better understand and predict turbulence quantities in the developing jet region, where most of the jet noise is produced. Comparisons were made with available PIV data collected at the SHJAR facility (ref. 4) at NASA Glenn Research Center.

The jet flowfields were computed using an axisymmetric 316x171 computational grid, which included the internal nozzle region well upstream of the exit plane. The computational domain extended 100 jet exit radii in the streamwise direction and 25 jet exit radii radially, sufficiently distant to minimize boundary condition placement impact on the shear layer entrainment path-lines.

Solution convergence was determined for all cases when the $\mathrm{L}_{2}$ of the residual for the eight transport equations dropped five orders of magnitude relative to the initial solution. Solution grid resolution insensitivity was verified using a computational mesh with twice the radial grid density and then comparing predicted centerline values between the coarse and fine grid solutions. For all cases, the comparisons were identical.

Nozzle flow boundary conditions were prescribed uniformly as inflow stagnation conditions for pressure and temperature imposed well upstream of the nozzle exit. For solution stability, values for turbulent kinetic energy, dissipation rate, and scalar variance were monitored throughout the flow domain for numerical exceptions; in such cases background "zero" levels (1e-16) were prescribed.

The boundary layer region is an important aspect of the nozzle flowfield and can impact the strength of the initial jet shear layer. However, accurately resolving the turbulence and mean flow strain characteristics through the laminar sublayer was beyond scope of the present effort. As a reasonable approximation, a compressible wall function formulation was used to analytically surface boundary conditions to points in the inertial region. Grid resolution is still required to resolve the momentum deficit of the boundary layer; approximately ten mesh points were provided within the boundary layer region near the nozzle exit. Additionally, all wall surfaces were assumed to be adiabatic.

Results for predicting the mean velocity and turbulent kinetic energy using the turbulence modeling framework under current study is discussed in detail in reference 3 for the subsonic cases listed in table II. Inclusion of the turbulent variance equations had no impact on the prediction of these quantities.

TABLE II.-TANNA MATRIX SET POINT CONDITIONS

\begin{tabular}{|c|l|l|l|l|l|}
\hline S.P. & NPR & $T_{t, j} / T_{\infty}$ & $T_{j} / T_{\infty}$ & $M_{a c}{ }^{*}$ & $M_{j}$ \\
\hline 03 & 1.197 & 1.000 & 0.950 & 0.50 & 0.51 \\
\hline 23 & 1.103 & 1.810 & 1.760 & 0.50 & 0.37 \\
\hline 42 & 1.066 & 2.750 & 2.700 & 0.50 & 0.30 \\
\hline 07 & 1.861 & 1.000 & 0.840 & 0.90 & 0.98 \\
\hline 27 & 1.361 & 1.922 & 1.760 & 0.90 & 0.68 \\
\hline 46 & 1.225 & 2.861 & 2.700 & 0.90 & 0.54 \\
\hline 29 & 1.900 & 2.114 & 1.760 & 1.33 & 1.00 \\
\hline 49 & 1.692 & 3.138 & 2.700 & 1.48 & 0.90 \\
\hline
\end{tabular}

\section{Jet Scalar Variance Flowfield Results}

Comparison of the resulting static and temperature fluctuations using respectively the energy and total temperature variants of the scalar variance equations are shown in figures 2 and 3 As shown, at a given exit velocity (i.e., constant acoustic Mach number $M_{a c}$ ), the peak values for both the total and static temperature fluctuations increase with exit temperature. For the unheated jets (such as SP03 and SP07), static temperature fluctuations increase with jet exit velocity. The variance production term is influenced only by the magnitude of the temperature gradient. A drop in exit static temperature with increasing velocity results in larger density gradient, which in turn enhances the peak temperature variance. Use of a total temperature variance equation remedies this issue since the SP03 and SP07 cases are isoenergetic. It is worth noting that the peak total enthalpy also grows slowly with increased jet exit velocity due to turbulent viscous dissipation effects on the total enthalpy (see ref. 15). For 
example, for a turbulent round jet, the energy equation can be rewritten in terms of the total enthalpy equation with a source term proportional to the local velocity gradient as

$$
\frac{D}{D t}\left(\rho h_{t}\right)-\frac{\partial}{\partial r}\left(\alpha_{t} \frac{\partial h_{t}}{\partial r}\right) \approx \frac{\partial}{\partial r}\left(\left(\mu_{t}-\alpha_{t}\right) U \frac{\partial U}{\partial r}\right)
$$

For supersonic jet cases, differences in peak magnitudes for the static and total temperature variance are even more significant. Table III summarizes the series of unheated supersonic jets simulated during this study. Computational grids and nozzle geometries were supplied by NASA Glenn personnel for this effort. As shown in the comparisons of figure 4 , the peak centerline static temperature fluctuation can exceed $5 \%$ of the exit temperature for the Mach 1.8 case, whereas the peak total temperature fluctuations remain small, which is desired in jet noise predictions within the current framework. The computational assessment studies support the use of total temperature variance from RANS flowfield simulations for use in improving jet noise predictions in heated jets.

TABLE III.-FLOW CONDITIONS FOR

SUPERSONIC UNHEATED JET STUDIES

\begin{tabular}{|c|c|c|}
\hline \multicolumn{3}{|c|}{$\left(T_{t, j}=294.44 K\right)$} \\
\hline$M_{j}$ & NPR & $T_{j} / T_{\infty}$ \\
\hline 1.185 & 2.363 & 0.781 \\
\hline 1.400 & 3.162 & 0.718 \\
\hline 1.500 & 3.647 & 0.690 \\
\hline 1.660 & 4.618 & 0.645 \\
\hline 1.800 & 5.708 & 0.607 \\
\hline
\end{tabular}

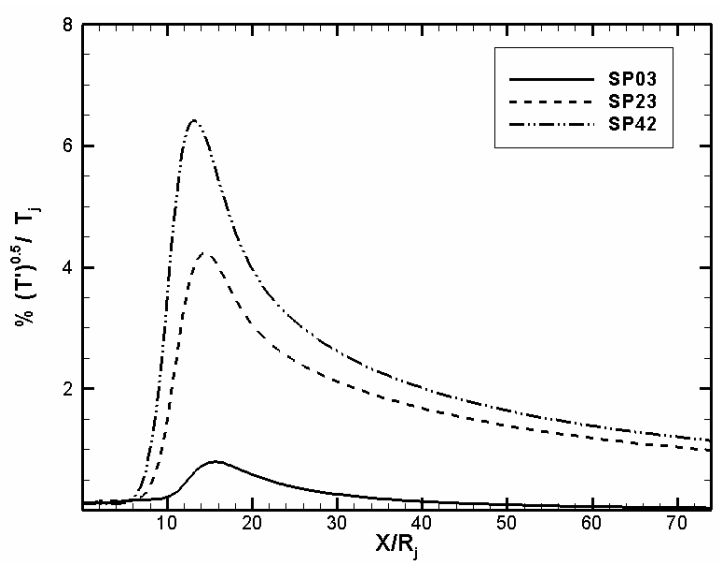

(a). $M_{a c}=0.50$

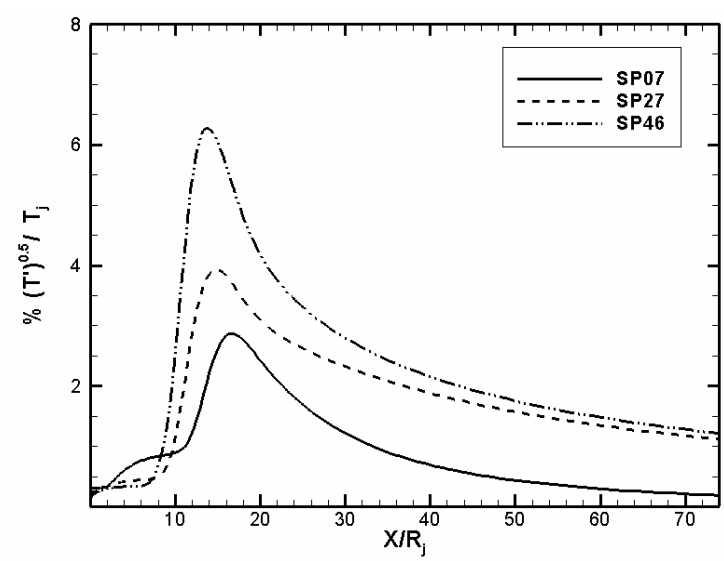

(b). $M_{a c}=0.90$

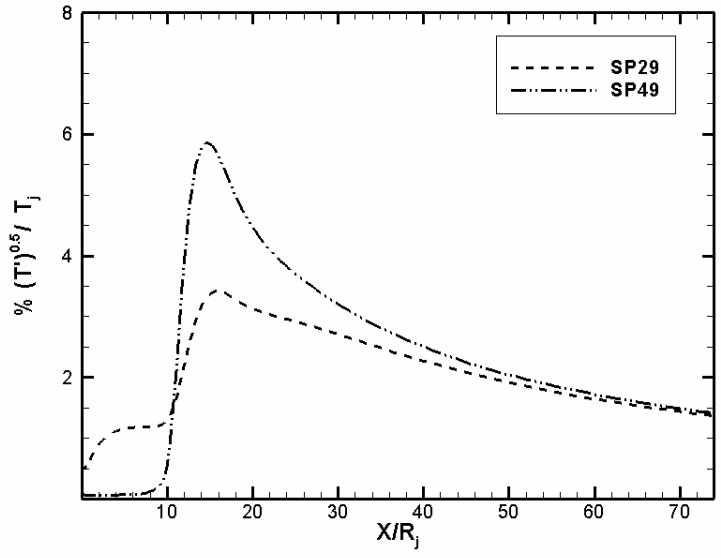

(c). $M_{a c}=1.33$ and $M_{a c}=1.48$

Figure 2.-Predicted temperature fluctuations using energy variance model. 


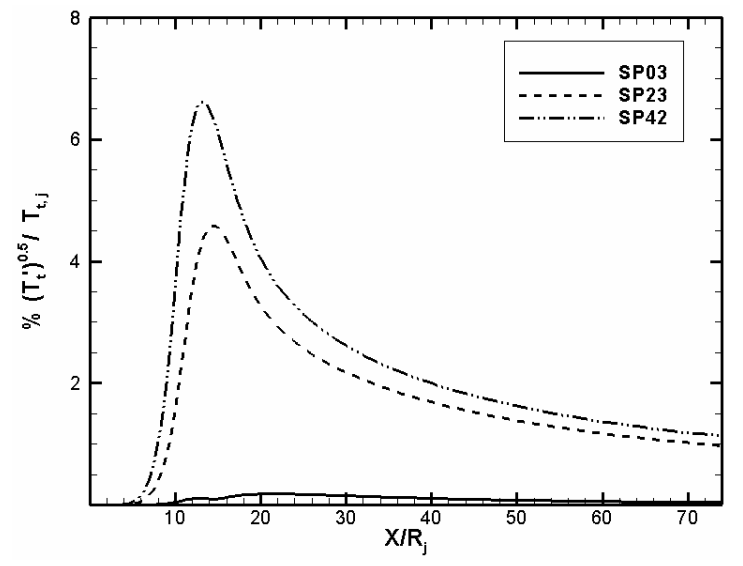

(a) $M_{a c}=0.50$

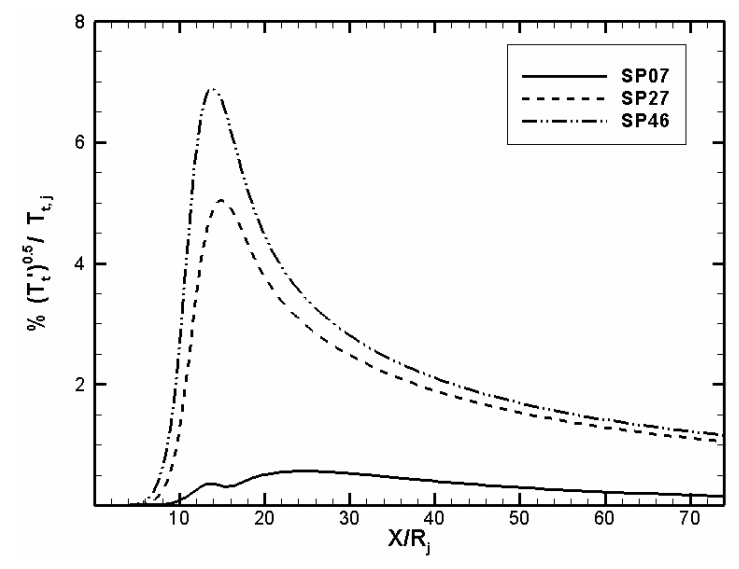

(b) $M_{a c}=0.90$

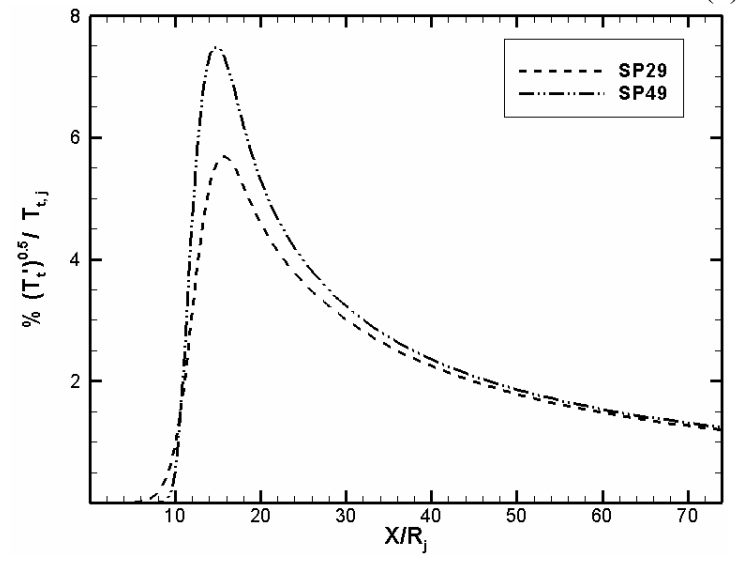

(c) $M_{a c}=1.33$ and $M_{a c}=1.48$

Figure 3.-Predicted temperature fluctuations using total enthalpy variance model.

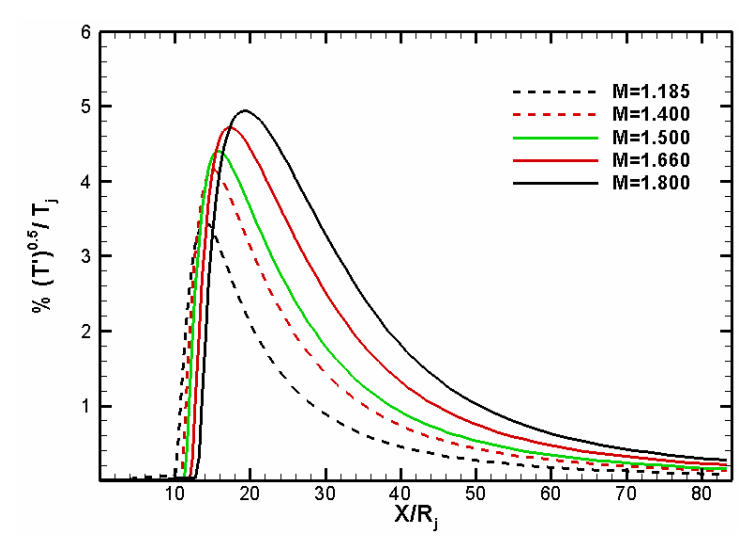

(a) Energy variance model

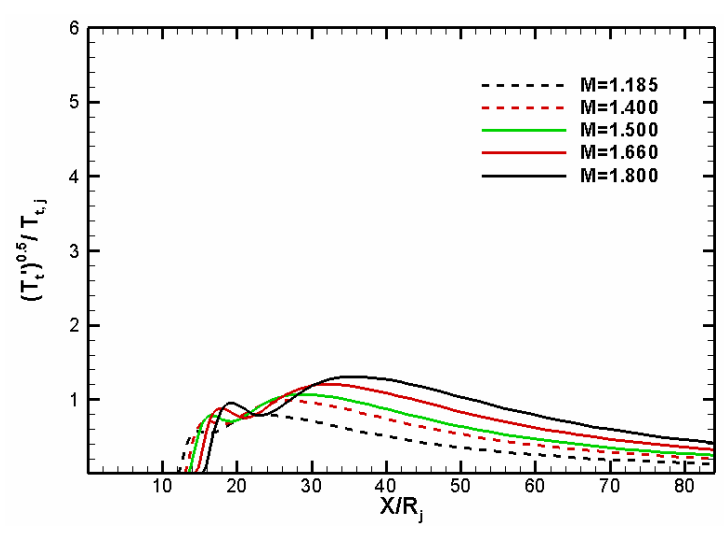

(b) Total enthalpy variance model

Figure 4.-Predicted temperature fluctuations for unheated supersonic jets. 


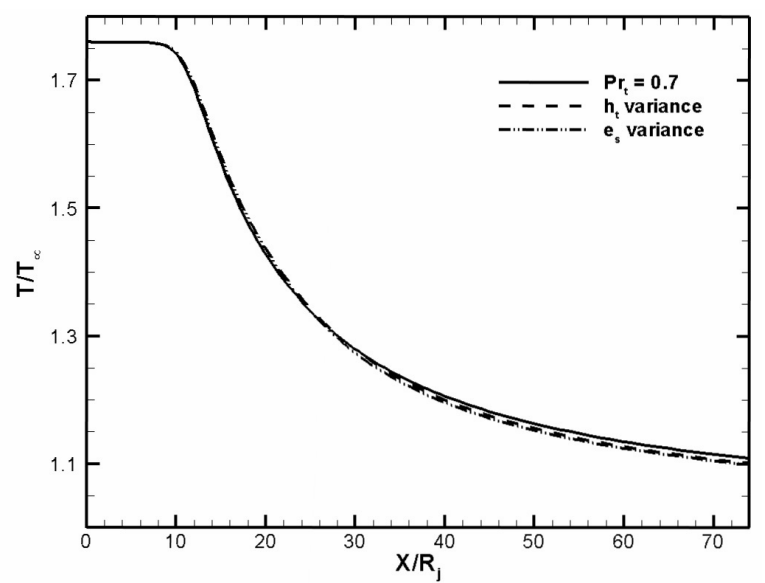

Figure 5.-Predicted centerline temperature decay comparison for SP27 using variable turbulent Prandtl number model.

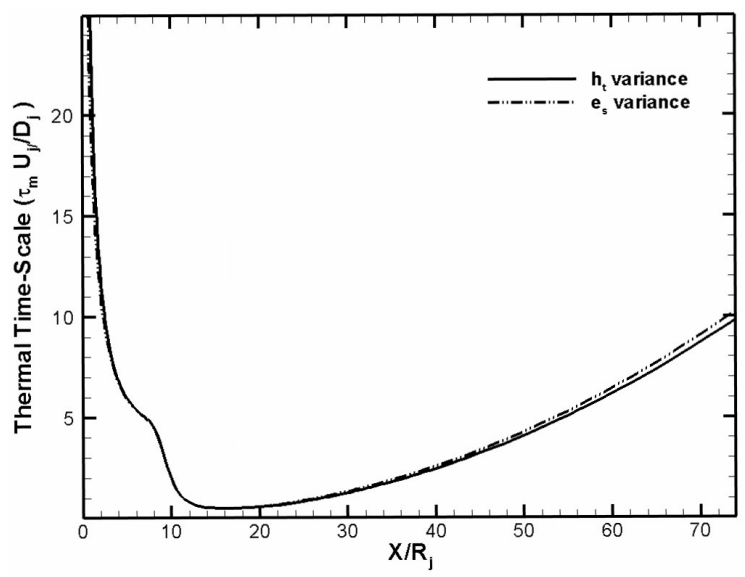

Figure 6.-Comparison of predicted thermal eddy timescales using total enthalpy vs. energy variance model: SP27 case.

\section{Thermal Mixing Using Total Enthalpy Fluctuations}

The baseline turbulent Prandtl number model utilizes local temperature variance (later extended to energy variance) and its dissipation rate to calculate turbulent thermal diffusivity. To determine the impact on using the total enthalpy variance for thermal mixing predictions, the SP27 low-speed heat jet was simulated using both modeling approaches. For comparison, the jet was also simulated using a constant turbulent Prandtl number of 0.7. As shown in figure 5, the centerline thermal mixing as predicted by the total enthalpy fluctuations is similar to that predicted by the baseline formulation. For both cases, the predicted total kinetic energy is the same since use of the variance model does not impact this quantity. Figure 6 compares the predicted thermal time scales using both variance models and shows similar agreement. Based on eq. (13), the predicted turbulent thermal diffusivity should therefore be similar.

\section{Jet Noise Predictions}

Spectral predictions presented here are for $\theta=90^{\circ}$ observer angle and are based on equations (B5) and (B6) discussed in the appendix. Numerical results are shown for all eight subsonic test points described in table II, and are compared with the lossless narrow-band data of reference 4 at a distance of $R / D_{j}=100$. In addition to the standard flow variables, which include the turbulent kinetic energy and time- and length-scales, this modified version of the flow solver permitted input of the local total temperature variance as predicted by the modified scalar variance model. Figure 7 shows that at the unheated conditions of SP03 and SP07 self-noise component dominates. With the addition of heat, the enthalpy-related noise overwhelms the self-noise at the static temperature ratio of 1.76 and 2.70; and increasingly more so at the higher temperature. As is pointed out in appendix B, outside the usual calibration constants $C_{\ell}$ and $C_{\tau}$ that are related to length- and time-scales, the final calibration of eqs. (B5) and (B6) requires a pair of constant $A$ and $B$. The predictions appear quite encouraging and show a consistent level for power spectral density at all test conditions examined here.

As a cautionary remark, due to the differences in the RANS predictions between various flow solvers, specifically in quantities such as turbulent kinetic energy and its dissipation rate, and probably the variance in total temperature, it in highly unlikely to define a universal set of constants for the above four calibration parameters. The final calibration thus becomes dependent on the specific RANS solver. 


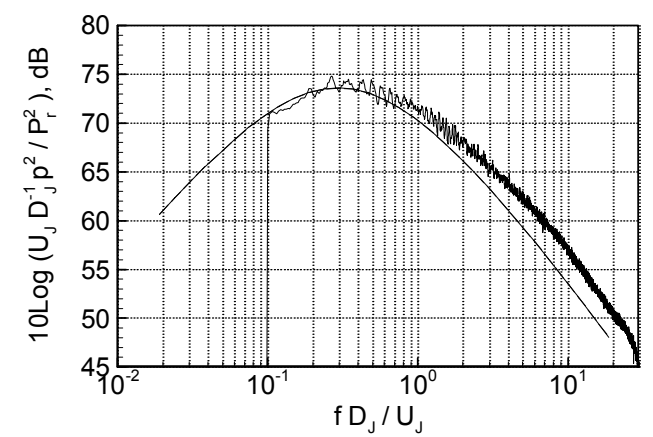

(a) $\mathrm{SP} 03$

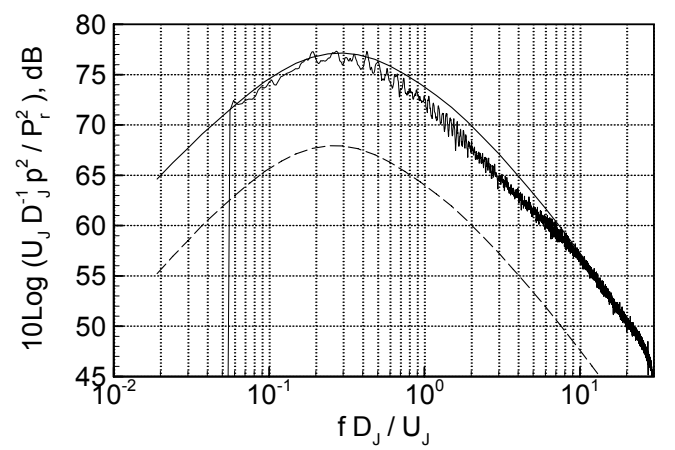

(c) SP23

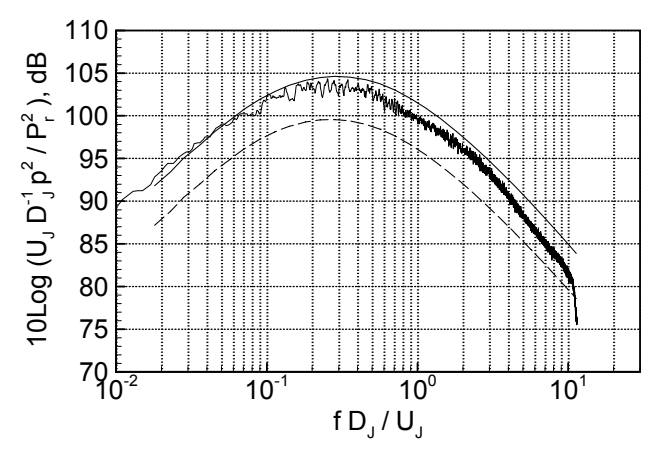

(e) SP29

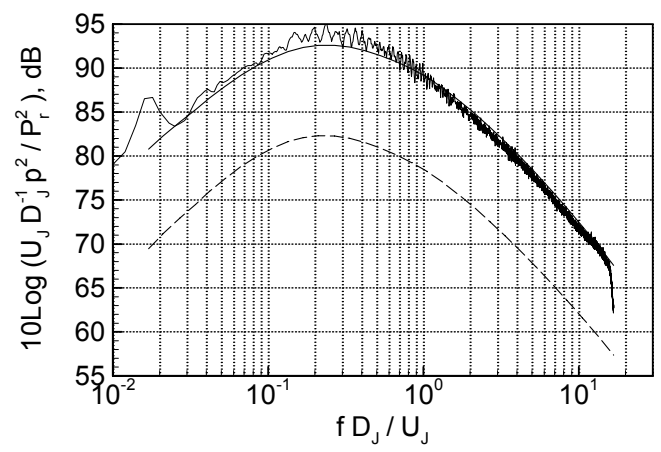

(g) SP46

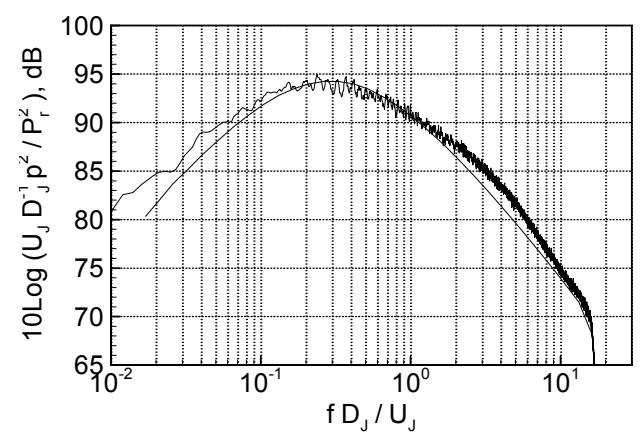

(b) SP07

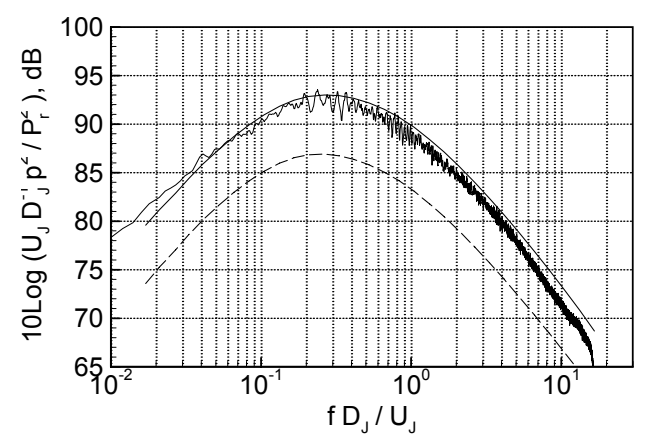

(d) SP27

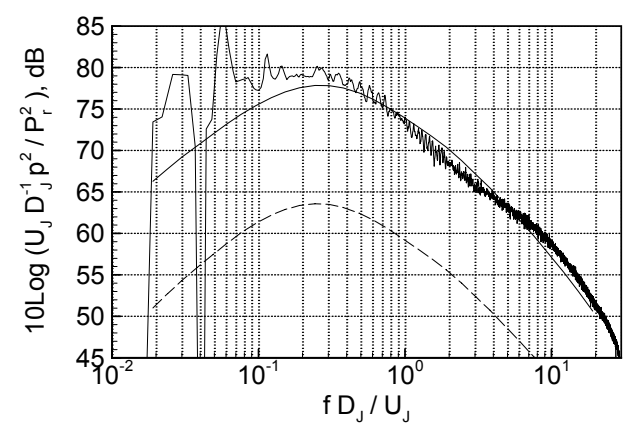

(f) SP42

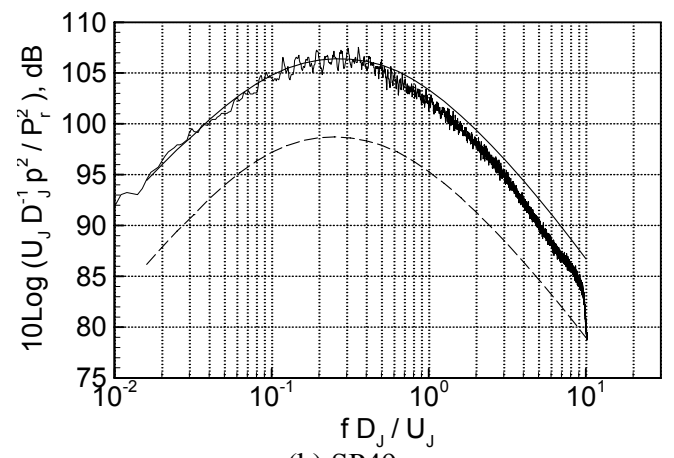

(h) SP49

Figure 7.-Predicted jet noise (lossless) comparisons at $90^{\circ}$ self-noise only (dashed) and self-noise + enthalpy-noise (solid). 


\section{Conclusions}

An acoustic analogy methodology has been presented in order to improve the far-field noise predictions in subsonic heated jets. The governing acoustic equation is an inhomogeneous Pridmore-Brown equation that is constructed from a linearized set of Euler equations when the base flow is represented as a locally parallel mean flow. The resulting nonlinear terms, all second-order in fluctuating variables, are identified as the equivalent sources of sound and are modeled.

The effect of heat on noise is attributed primarily to a fluctuating velocity/enthalpy source term. In the absence of any experimental measurements, a number of simplifying assumptions are required to model this new source. A specialized RANS solver supplies the variance in total enthalpy in addition to the standard parameters. This flow solver uses a pair of transport equations, governing the variance in total enthalpy and its dissipation rate, coupled with the standard two-equation $k-\varepsilon$ model.

Sample $90^{\circ}$ spectral computations demonstrate that the boost in source strength is sufficient to correct the earlier deficits predicted in the absence of the enthalpy source term. Outside the two calibration parameters related to timeand length-scales of turbulence velocity fluctuations, the final calibration is achieved when two additional constants are introduced that amount to using a linear combination of the two sources. This is required partially due to the neglect of the cross-correlation between various source terms, as well as differences in scales between velocity and thermal fluctuations.

Extension of the above study to supersonic jets is currently underway. Preliminary RANS solutions indicate some level of sensitivity in the predicted turbulent kinetic energy with/without compressibility corrections. These corrections are normally introduced to predict a better mean flow, and their impact on turbulence requires further consideration. 



\section{Appendix A-Source Model}

The far-field sound spiral density per unit volume of turbulence at $\vec{y}$ is

$$
\overline{p^{2}}(\vec{x}, \vec{y}, \omega)=|G(\vec{x}, \vec{y}, \omega)|^{2} \int_{\vec{\xi}}^{+\infty} \int_{-\infty}^{+\infty} q(\vec{y}, \vec{\xi}, \tau) e^{i \omega \tau} e^{-i \vec{k} \cdot \vec{\xi}} d \tau d \vec{\xi},
$$

where $q$ denotes a two-point space-time correlation between fluctuating variables at source points A and B separated by space $\vec{\xi}$ and time $\tau$, and $G$ is the relevant Green's function (ref. 16). For simplicity, we consider the turbulence as isotropic as described by Batchelor (ref. 17), and describe a two-point space-time correlation of the velocity components (notation $u_{i}$ is now used in place of $v_{i}^{\prime}$ as a fluctuating velocity component.)

$$
\begin{gathered}
\overline{\left(u_{i}\right)_{A}\left(u_{j}\right)_{B}}=\overline{u^{2}}\left[\left(f+\frac{1}{2} \xi f^{\prime}\right) \delta_{i j}-\frac{1}{2} \frac{\xi_{i} \xi_{j}}{\xi} f^{\prime}\right], \\
f^{\prime}(\xi, \tau) \equiv \frac{\partial f}{\partial \xi}, \quad \xi^{2}=\xi_{1}^{2}+\xi_{2}^{2}+\xi_{3}^{2} .
\end{gathered}
$$

Consider a fourth-order time-delayed space-time correlation per unit volume of turbulence at $\vec{y}$

$$
R_{i j k \ell}(\vec{y}, \vec{\xi}, \tau) \equiv \overline{u_{i} u_{j} u_{k}^{\prime} u_{\ell}^{\prime}} .
$$

The axial component of the above tensor is

$$
R_{1111}(\vec{y}, \vec{\xi}, \tau)=\left(\overline{u_{1}^{2}}\right)^{2} H(\vec{\xi}, \tau),
$$

and its spectral density is the four-dimensional wave-number frequency spectrum function

$$
I_{1111}\left(\vec{y}, \omega^{s}\right)=\int_{\vec{\xi}} \int_{\tau} R_{1111}(\vec{y}, \vec{\xi}, \tau) e^{i \omega^{s} \tau} e^{-i \vec{k} \cdot \vec{\xi}} d \tau d \vec{\xi} .
$$

The above integral was written in a frame moving with source convection velocity $U_{c}$, and $\omega^{\mathrm{s}}$ is the source frequency in that frame and is related to the observer frequency through the Doppler factor. If turbulence correlation coefficients are quasi normal, then the axial component of the above tensor becomes $\overline{u_{1} u_{1} u_{1}^{\prime} u_{1}^{\prime}}=2\left(\overline{u_{1} u_{1}^{\prime}}\right)^{2}$ and subsequently

$$
I_{1111}\left(\vec{y}, \omega^{s}\right)=2\left(\bar{u}_{1}^{2}\right)^{2} \int_{\vec{\xi}} \int_{\tau}\left[\left(f+\frac{1}{2} \xi f^{\prime}\right)-\frac{1}{2} \frac{\xi_{1} \xi_{1}}{\xi} f^{\prime}\right]^{2} e^{i \omega^{s} \tau} e^{-i \vec{k} \cdot \vec{\xi}} d \tau d \vec{\xi} .
$$

Similarly, consider the two point space time correlation for the enthalpy/velocity source term

$$
\begin{gathered}
\Im_{i j}(\vec{y}, \vec{\xi}, \tau) \equiv \overline{\left(u_{i} \frac{h_{t}^{\prime}}{\tilde{h}}\right)_{A}\left(u_{j} \frac{h_{t}^{\prime}}{\tilde{h}}\right)_{B}} \cong \frac{1}{\tilde{h}^{2}} \overline{\left(u_{i} h_{t}^{\prime}\right)_{A}\left(u_{j} h_{t}^{\prime}\right)_{B}}, \\
\Xi_{i j}\left(\vec{y}, \omega^{s}\right) \equiv \int_{\vec{\xi} \tau} \int_{\tau} \Im_{i j}(\vec{y}, \vec{\xi}, \tau) e^{i \omega^{s} \tau} e^{-i \vec{k} . \vec{\xi}} d \tau d \vec{\xi} .
\end{gathered}
$$

Using Millionshtchikov's hypothesis (ref. 17) and noting that the autocorrelation of $\left(u_{i} h_{t}^{\prime}\right)$ is zero in isotropic turbulence, 


$$
\overline{\left(u_{i} h_{t}^{\prime}\right)_{A}\left(u_{j} h_{t}^{\prime}\right)_{B}}=\overline{\left(u_{i}\right)_{A}\left(u_{j}\right)_{B}} \overline{\left(h_{t}^{\prime}\right)_{A}\left(h_{t}^{\prime}\right)_{B}} .
$$

Now if the space/time functions governing the enthalpy fluctuations decay in a similar fashion as that of the velocity fluctuations then

$$
\Xi_{11}\left(\vec{y}, \omega^{s}\right)=\frac{\overline{\left(h_{t}^{\prime}\right)^{2}}}{\tilde{h}^{2}} \overline{u_{1}^{2}} \int_{\vec{\xi}} \int_{\tau}\left[\left(f+\frac{1}{2} \xi f^{\prime}\right)-\frac{1}{2} \frac{\xi_{1} \xi_{1}}{\xi} f^{\prime}\right]^{2} e^{-i \vec{k} \cdot \vec{\xi}} e^{i \omega^{s} \tau} d \tau d \vec{\xi}
$$

or

$$
\Xi_{11}\left(\vec{y}, \omega^{s}\right)=\frac{1}{2} \frac{1}{\overline{u_{1}^{2}}} \frac{\overline{\left(h_{t}^{\prime}\right)^{2}}}{\tilde{h}^{2}} I_{1111}
$$

Following (ref. 16) we assume

$$
f(\xi, \tau)=\exp \left(-\frac{\pi \xi}{\ell}-\left|\tau / \tau_{o}\right|\right)
$$

then

$$
I_{1111}=\frac{4 \ell^{3}}{5 \pi^{2}}\left(\overline{u_{1}^{2}}\right)^{2} H\left(\omega^{s}\right) N(k \ell) ; \quad H\left(\omega^{s}\right)=\int_{-\infty}^{+\infty} \exp \left(-2\left|\frac{\tau}{\tau_{o}}\right|+i \omega^{s} \tau\right) d \tau=\frac{\tau_{o}}{1+\left(\omega^{s} \tau_{o} / 2\right)^{2}}
$$

where $N(k \ell)$ is a non-compactness factor (ref. 18).

In general, if the thermal eddy time-scale $\tau_{1}$ is different from the turbulence time-scale $\tau_{o}$ then the following Fourier transform

$$
H\left(\omega^{s}\right)=\int_{-\infty}^{+\infty} \exp \left(-\left|\frac{\tau}{\tau_{o}}\right|-\left|\frac{\tau}{\tau_{1}}\right|+i \omega^{s} \tau\right) d \tau=\frac{\tau^{*}}{1+\left(\omega^{s} \tau^{*} / 2\right)^{2}}, \quad \tau^{*} \equiv \frac{2 \tau_{o} \tau_{1}}{\tau_{o}+\tau_{1}}
$$

is used in the velocity/enthalpy spectrum function (A11). 


\section{Appendix B-Source Green's Function Convolution}

A stationary point source with frequency $\omega$ and location $\vec{x}^{s}$ (superscript $s$ denotes a source location) is considered in defining the Green's Function (GF)

$$
L\left(\mathbf{G} e^{-i \omega t}\right)=c_{\infty}^{2} e^{-i \omega t} \delta\left(\vec{x}-\vec{x}^{s}\right),
$$

and

$$
\mathbf{G}\left(\vec{x}, \vec{x}^{s}, \omega\right)=\frac{1}{4 \pi \omega R} e^{i k\left(R-x_{1}^{s} \cos \theta\right)} \sum_{m=0}^{\infty} f^{m}\left(r^{s}, k, \theta\right) \cos m\left(\varphi-\varphi^{s}\right),
$$

where $f^{m}\left(r^{s}, k, \theta\right)$ is a solution to second-order compressible Rayleigh operator (ref. 16), corresponding to mode $m$ ( $m$ is not a power), wave number $k=\omega / c_{\infty}$ (to avoid confusion, notation $\kappa$ will be used to denotes TKE in this appendix), and observer angle $\theta$. Superscript $s$ denotes a source location.

The relevant GF associated with a moving singularity with source frequency $\omega^{\mathrm{s}}$ and convection velocity $\hat{i} U_{c}$ is

$$
\begin{gathered}
L\left(G e^{-i \omega t}\right)=\frac{D}{D t}\left[c_{\infty}^{2} e^{-i \omega^{s} t} \delta\left(x_{1}-U_{c} t\right) \delta\left(\vec{x}_{t}-\vec{x}_{t}^{s}\right)\right], \\
G\left(\vec{x}, \vec{x}^{s}, \omega\right)=\frac{-i}{4 \pi R} \frac{\left(1-M^{s} \cos \theta\right)}{\left(1-M_{c} \cos \theta\right)} e^{i k R} \sum_{m} f^{m}\left(r^{s}, k, \theta\right) \cos m\left(\varphi-\varphi^{s}\right) .
\end{gathered}
$$

We make the simplifying assumption that the source components described in Eq. (7) are uncorrelated; further we consider the following two relatively more important source terms at $90^{\circ}$

$$
\begin{gathered}
D \frac{\partial^{2} u_{i} u_{j}}{\partial x_{i} \partial x_{j}} \\
\text { Self }
\end{gathered} \quad D^{2} \frac{\partial}{\partial x_{j}}\left(u_{j} \frac{h_{t}^{\prime}}{\tilde{h}}\right)
$$

As usual, the spatial derivatives are moved from source to the Green's function. For example

$$
\mathbf{G} \frac{\partial^{2} u_{i} u_{j}}{\partial x_{i} \partial x_{j}} \rightarrow u_{i} u_{j} \frac{\partial^{2} \mathbf{G}}{\partial x_{i} \partial x_{j}}
$$

Some level of simplification is achieved when we consider components of turbulence fluctuations in the direction of the observer $\theta$ as suggested by Proudman (ref. 19). The above sources of interest would now be designated as $D \frac{\partial^{2} u_{\eta} u_{\eta}}{\partial \eta \partial \eta}$ and $D^{2} \frac{\partial}{\partial \eta}\left(u_{\eta} \frac{h_{t}^{\prime}}{\tilde{h}}\right)$ where $\eta$ is the spatial separation of the correlation in the direction of the observer (see sketch).

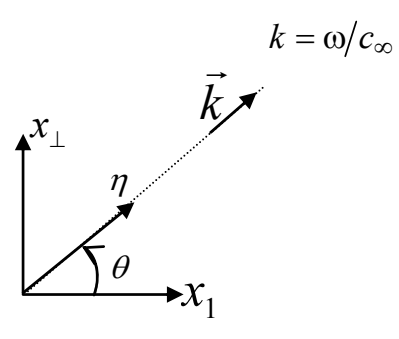


The required spatial derivatives of the Green's function are

$$
\begin{aligned}
& \mathbf{G}_{, \eta}=\cos \theta \mathbf{G}_{, 1}+\sin \theta \mathbf{G}_{, r} \\
& \mathbf{G}_{, \eta \eta}=\cos ^{2} \theta \mathbf{G}_{, 11}+\sin ^{2} \theta \mathbf{G}_{, r r}+2 \sin \theta \cos \theta \mathbf{G}_{, 1 r}
\end{aligned}
$$

where $\mathbf{G}_{, 1}=-i k \cos \theta \mathbf{G}$ and the radial derivative $\mathbf{G}_{, r}$ applies to the function $f^{m}(r, k, \theta)$.

The following simplifications are introduced into the radial derivatives using the high frequency solution to the GF

$$
f_{, r}^{m} \cong-i k Q f^{m}, \quad f_{, r r}^{m} \cong-(k Q)^{2} f^{m},
$$

where

$$
Q^{2} \equiv \frac{\rho^{s}}{\rho_{\infty}}\left(1-M^{s} \cos \theta\right)^{2}-\cos ^{2} \theta .
$$

A more accurate representation of function $Q$ should account for mode number

$$
Q_{m}^{2} \equiv \frac{\rho^{s}}{\rho_{\infty}}\left(1-M^{s} \cos \theta\right)^{2}-\cos ^{2} \theta-\left(\frac{m}{k r}\right)^{2} .
$$

Since the zero ${ }^{\text {th }}$ mode usually makes the major contribution to the GF, the last term on the RHS was neglected in approximating the derivatives of the GF.

Subsequently, the following expression are derived for the far-field spectral density at $90^{\circ}$ per unit volume ringsource at $\vec{y}$

$$
\begin{aligned}
& {\overline{p^{2}}}_{\text {self }}(\vec{x}, \vec{y}, \omega) \cong\left(\frac{1}{4 \pi R}\right)^{2}\left(\frac{\rho^{s}}{\rho_{\infty}}\right)^{2}\left(\rho_{\infty}^{2} I_{\eta \eta \eta \eta}\right) k^{4} \sum_{m=0}^{\infty}\left(1+\delta_{m 0}\right) f^{m} f^{m^{*}}, \\
& {\overline{p^{2}}}_{\text {enthalpy }}(\vec{x}, \vec{y}, \omega) \cong\left(\frac{1}{4 \pi R}\right)^{2}\left(\frac{\rho^{s}}{\rho_{\infty}}\right)\left(c_{\infty}^{2} \rho_{\infty}^{2} \Xi_{\eta \eta}\right) k^{4} \sum_{m=0}^{\infty}\left(1+\delta_{m 0}\right) f^{m} f^{m^{*}},
\end{aligned}
$$

and

$$
\begin{aligned}
& \Xi_{\eta \eta}\left(\vec{y}, \omega^{s}\right)=\frac{1}{2} \frac{1}{\overline{u_{1}^{2}}} \frac{\overline{\left(h_{t}^{\prime}\right)^{2}}}{\tilde{h}^{2}} I_{\eta \eta \eta \eta}, \quad \overline{u_{1}^{2}}=\frac{2 \kappa}{3}, \\
& I_{\eta \eta \eta \eta}\left(\vec{y}, \omega^{s}\right)=\kappa^{7 / 2} \frac{\tau_{o}^{4}}{1+\left(\omega^{s} \tau_{o} / 2\right)^{2}} N(k \ell) .
\end{aligned}
$$

The turbulence length- and time-scales are calculated the usual way from the turbulent kinetic energy and its dissipation rate as $\ell=C_{\ell} \kappa^{1.5} / \varepsilon$ and $\tau_{o}=C_{\tau} \kappa / \varepsilon$. The final calibration is achieved when the above noise components (B5) and (B6) are also multiplied by constant parameters $A$ and $B$ respectively. 


\section{References}

1 Khavaran, A., Bridges, J. and Georgiadis, N., "Prediction of Turbulence-Generated Noise in Unheated Jets, Part 1: JeNo Technical Manual (Version 1.0)," NASA/TM-2005-213827, July 2005.

2 Tanna, H.K., "The Influence of Temperature on Shock-Free Supersonic Jet Noise," J. Sound Vib., 39(4), pp. 429-460, 1975.

3 Kenzakowski, D.C., "Turbulence Modeling Improvements for Jet Noise Prediction Using PIV Datasets," $10^{\text {th }}$ AIAA/CEAS Aeroacoustics Conf., AIAA-2004-2978.

4 Bridges, J. and Wernet, M., "Measurements of the Aeroacoustic Sound Source in Hot Jets," $9^{\text {th }}$ AIAA/CEAS Aeroacoustics Conf., AIAA-2003-3130.

5 Viswanathan, K., “Aeroacoustics of Hot Jets,” J. Fluid Mechanics, 519, pp.39-82, 2004.

6 Tam, C.K.W., "Dimensional Analysis of Jet Noise Data," AIAA-2005-2938, 2005.

7 Morfey, C.L., and Szewczyk, V.M., "New Scaling Laws for Hot and Cold Jet Mixing Noise Based on a Geometric Acoustic Model, “ J. Sound Vib., 61(2), pp. 255-292, 1978.

8 Lush, P.A., and Fisher, M.J., AGARD CP-131 (Noise Mechanisms), Chapter 12: Noise from Hot Jets, 1973.

9 Lilley, G.M., "The Radiated Noise from Isotropic Turbulence with Application to the Theory of Jet Noise," J. Sound Vib., 190(3), pp. 463-476, 1996.

10 Goldstein, M.E., “A Generalized Acoustic Analogy,” J. Fluid Mechanics, 488, pp. 315-333, 2003.

11 Kenzakowski, D.C., "RANS Modeling Improvements for Jets Using Scalar Variance Equations, AIAA-20060491.

12 Nagano, Y. and Kim. C., “A Two-Equation Model for Heat Transport in Wall Turbulent Shear Flows,” J. Heat Transfer, (110), pp. 583-589, 1988.

13 Goldstein, M.G., Khavaran, A., and Musafir, R.E., "Jet Noise Predictions Based on Two Different forms of Lilley's Equation," NASA/TM-2005-213829 (Part 1 and 2), 2005.

14 Chidambaram N., Dash, S.M., and Kenzakowski, D.C., "Scalar Variance Transport in the Turbulence Modeling of Propulsive Jets," J. Propulsion \& Power, Jan-Feb., 2001.

15 Tannehill, J., Anderson, D., and Pletcher, R., "Computational Fluid Dynamics and Heat Transfer," $2^{\text {nd }}$ ed., 1997.

16 Khavaran, A., N. J., Bridges, J. E., and Dippold, V. F., "Effect of Free Jet on Refraction and Noise," AIAA2005-2941, 2005.

17 Batchelor, G.K., The Theory of Homogeneous Turbulence, Cambridge University Press, 1953.

18 Khavaran, A., and Bridges, J. E., "Modeling of Turbulence Generated Noise in Jets," AIAA-2004-2983, 2004.

19 Proudman, I., "The Generation of Noise by Isotropic Turbulence," The Proceeding of the Royal Society of London, A214, pp. 119-132, 1952. 


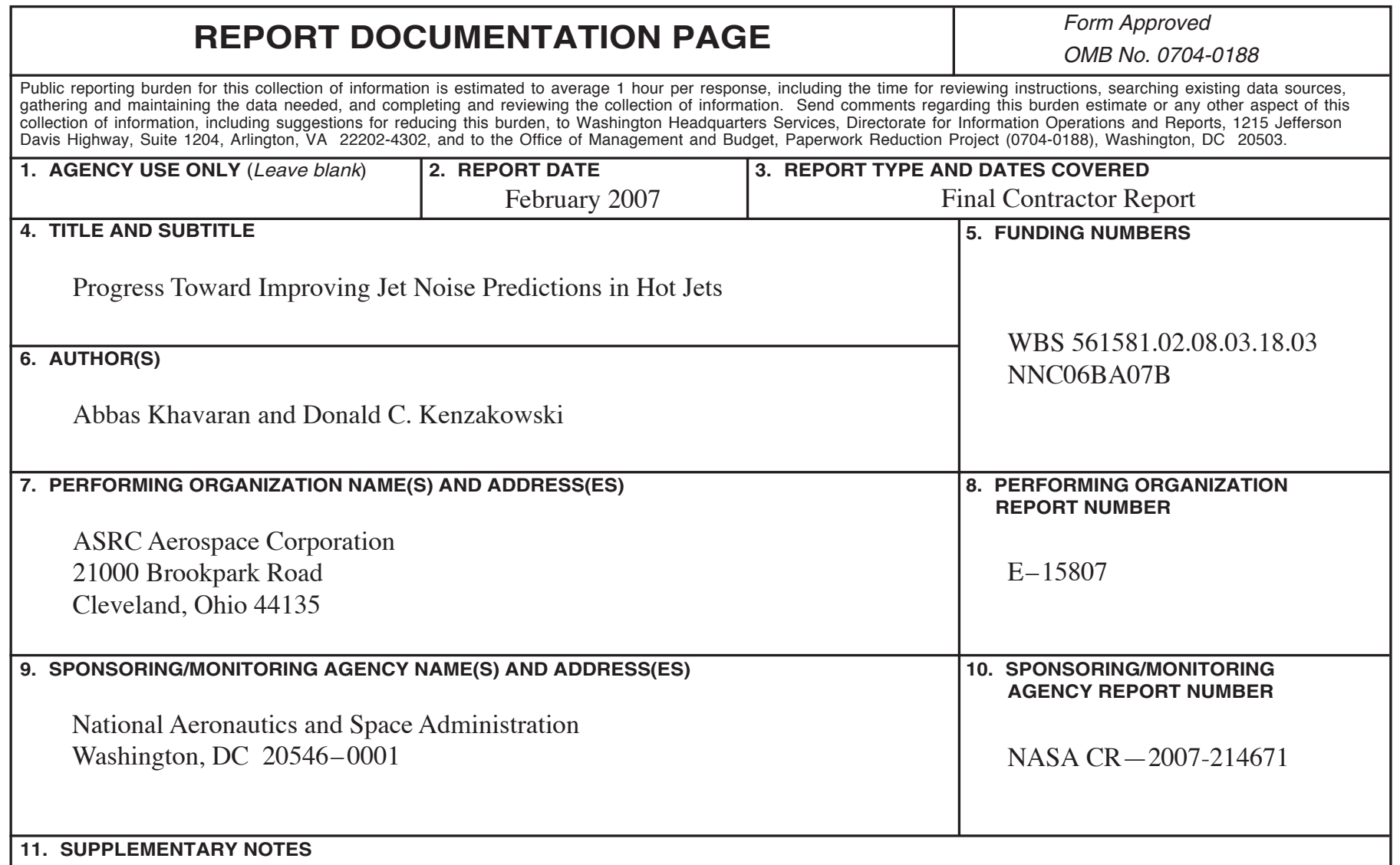

Abbas Khavaran, ASRC Aerospace Corporation, 21000 Brookpark Road, Cleveland, Ohio 44135, e-mail:

khavaran@grc.nasa.gov; and Donald C. Kenzakowski, e-mail: kenzakow@craft-tech.com, Combustion Research \& Flow Technology, Inc. (CRAFT Tech), 6210 Keller's Church Rd., Pipersville, Pennsylvania 18947. Responsible person, Abbas Khavaran, organization code RTA, 216-977-1120.

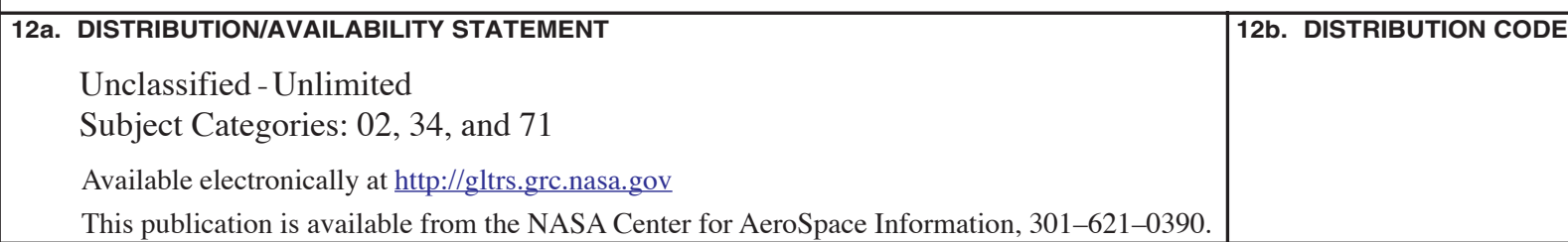

\section{ABSTRACT (Maximum 200 words)}

An acoustic analogy methodology for improving noise predictions in hot round jets is presented. Past approaches have often neglected the impact of temperature fluctuations on the predicted sound spectral density, which could be significant for heated jets, and this has yielded noticeable acoustic under-predictions in such cases. The governing acoustic equations adopted here are a set of linearized, inhomogeneous Euler equations. These equations are combined into a single thirdorder linear wave operator when the base flow is considered as a locally parallel mean flow. The remaining second-order fluctuations are regarded as the equivalent sources of sound and are modeled. It is shown that the hot jet effect may be introduced primarily through a fluctuating velocity/enthalpy term. Modeling this additional source requires specialized inputs from a RANS-based flowfield simulation. The information is supplied using an extension to a baseline twoequation turbulence model that predicts total enthalpy variance in addition to the standard parameters. Preliminary application of this model to a series of unheated and heated subsonic jets shows significant improvement in the acoustic predictions at the $90^{\circ}$ observer angle.

\section{SUBJECT TERMS}

Noise; Jet noise; Propulsion noise; Flow noise

\begin{tabular}{|c|c|c|c|}
\hline $\begin{array}{c}\text { 17. SECURITY CLASSIFICATION } \\
\text { OF REPORT } \\
\text { Unclassified }\end{array}$ & $\begin{array}{c}\text { 18. SECURITY CLASSIFICATION } \\
\text { OF THIS PAGE } \\
\text { Unclassified }\end{array}$ & $\begin{array}{c}\text { 19. SECURITY CLASSIFICATION } \\
\text { OF ABSTRACT } \\
\text { Unclassified }\end{array}$ \\
\hline
\end{tabular}



